\title{
Neural circuits in anxiety and stress disorders: a focused review
}

\author{
This article was published in the following Dove Press journal: \\ Therapeutics and Clinical Risk Management \\ 23 January 2015 \\ Number of times this article has been viewed
}

\section{Elizabeth R Duval \\ Arash Javanbakht \\ Israel Liberzon}

Department of Psychiatry, University of Michigan Health System, Ann Arbor, MI, USA
Correspondence: Elizabeth R Duval

Department of Psychiatry, University of Michigan Health System, 4250

Plymouth Rd, Ann Arbor, MI 48I09 USA

Tel + I 7349364397

Email eduval@med.umich.edu
Abstract: Anxiety and stress disorders are among the most prevalent neuropsychiatric disorders. In recent years, multiple studies have examined brain regions and networks involved in anxiety symptomatology in an effort to better understand the mechanisms involved and to develop more effective treatments. However, much remains unknown regarding the specific abnormalities and interactions between networks of regions underlying anxiety disorder presentations. We examined recent neuroimaging literature that aims to identify neural mechanisms underlying anxiety, searching for patterns of neural dysfunction that might be specific to different anxiety disorder categories. Across different anxiety and stress disorders, patterns of hyperactivation in emotion-generating regions and hypoactivation in prefrontal/regulatory regions are common in the literature. Interestingly, evidence of differential patterns is also emerging, such that within a spectrum of disorders ranging from more fear-based to more anxiety-based, greater involvement of emotion-generating regions is reported in panic disorder and specific phobia, and greater involvement of prefrontal regions is reported in generalized anxiety disorder and posttraumatic stress disorder. We summarize the pertinent literature and suggest areas for continued investigation.

Keywords: fear, anxiety, neuroimaging

\section{Introduction}

Anxiety and stress disorders are among the most prevalent categories of mental illnesses, with a median onset at age 11 years and a lifetime prevalence of $28 \% .{ }^{1}$ When left untreated, anxiety symptoms persist and are associated with significant impairments in functioning, poor quality of life, and a huge economic burden. ${ }^{2-4}$ Anxiety disorders are of particular importance in the context of recent and ongoing world conflicts, as environmental factors can have a strong impact on anxiety and stress disorder development, particularly posttraumatic stress disorder (PTSD). ${ }^{5}$ Given the high prevalence rates, negative effects on many aspects of functioning, and environmental factors associated with trauma and stress, it is imperative that we continue to improve our understanding of the mechanisms underlying anxiety disorder presentations in an effort to improve existing treatments.

Although anxiety disorders have been extensively studied, the literature examining underlying neural mechanisms remains scarce, with relatively little evidence identifying specific deficits for various anxiety disorders. Despite the lack of concrete knowledge regarding the specific mechanisms underlying anxiety, both pharmacologic (selective serotonin reuptake inhibitors) and psychotherapeutic (cognitive behavioral therapy) treatments for anxiety management have been developed. These treatments are effective for many patients suffering from anxiety, but the exact mechanisms of action are not well known. Moreover, many patients do not have access to or do not experience 
complete symptom relief with the existing evidence-based treatments. It is thus imperative that we continue striving for an improved understanding of the specific neural deficits underlying anxiety disorder presentations and the mechanisms of action by which effective treatments reduce anxiety symptomatology. The widely accepted categorization of anxiety disorders is based solely on behavioral and subjective experiences. However, it is possible that the neural mechanisms underlying anxiety symptomatology overlap across a number of discretely defined disorders. In fact, arguments are emerging that anxiety disorders may fall along a continuum ranging from specific fear-based reactivity to more diffuse and prolonged stress or apprehension. Identifying mechanisms will allow for more accurate diagnostic categories, improved ability to predict individual treatment response, and will provide specific targets for new and improved treatments aimed at correcting aberrant neuronal functioning.

\section{Clinical categorization of anxiety disorders}

According to the Diagnostic and Statistical Manual of Mental Disorders, 5th Edition, ${ }^{5}$ anxiety and stress disorders are characterized by an excessive fear response and/or worry that interferes with functioning or causes significant distress. This class of disorders includes panic disorder (PD), specific phobia (SP), social anxiety disorder (SAD), PTSD, and generalized anxiety disorder (GAD).

\section{Panic disorder}

PD is characterized by sudden panic attacks, often occurring unexpectedly, followed by a month or more of worrying about having another attack or the consequence of the attack (eg, heart attack, stroke). Common symptoms during a panic attack include racing heart, shortness of breath, tightness in chest, paresthesia, gastrointestinal distress, sweating, hot/cold flashes, fear of dying, and fear of losing control. PD sufferers often develop agoraphobia, avoiding places or situations where they think they might have a panic attack.

\section{Specific phobia}

SP is characterized by excessive fear triggered by a specific object or situation. SP falls into four subtypes, including animal, natural environment (eg, heights, storms), bloodinjection-injury, and other (choking, vomiting, illness, costumed characters, etc). The excessive fear brought on by the phobic object or situation leads to intense distress, anxious anticipation, panic attacks, and/or avoidance of the feared object or situation.

\section{Social anxiety disorder}

SAD is characterized by persistent fear of social or performance situations resulting from the possibility of negative judgment, embarrassment, or humiliation. Cognitive distortions and self-monitoring in social situations, involving hyperawareness of internal cues and behaviors, are often associated with SAD. Feared social situations are avoided or tolerated with dread.

\section{Posttraumatic stress disorder}

PTSD can develop after exposure to serious injury, death, or a potential threat to the physical integrity of self or others. Symptom clusters include intrusive reexperiencing symptoms (memories of trauma, nightmares, flashbacks); avoidance of trauma-related thoughts, memories, contexts or cues; negative mood and cognition; and hyperarousal/ hypervigilance.

\section{Generalized anxiety disorder}

The core symptom in GAD is excessive and continuous worry, anxiety, and apprehensive expectation in multiple contexts. The ambiguity and diversity of the sources of stress and anxiety distinguishes this disorder from cue-related anxiety disorders such as PD, SP, SAD, and PTSD. There is a cognitive component to GAD that is characterized by worrisome thoughts and cognitive errors.

\section{Fear versus anxiety}

An important but controversial distinction in the anxiety and stress disorder literature is between the constructs of fear and anxiety. Fear and anxiety have considerable overlap with respect to subjective, behavioral, physiological, and neurological characteristics. However, some key differences separate the two. Fear is typically defined as a phasic and abrupt fight-or-flight response accompanied by intense arousal in response to an immediate and identifiable threat. Alternatively, anxiety is often defined by a more prolonged state of tension, worry, and apprehension regarding uncertain, and potentially negative, future events. ${ }^{6}$ Although fear and anxiety serve important evolutionary functions to keep us safe, fear allows us to combat or avoid immediate threats or danger, whereas anxiety increases vigilance and improves our ability to identify uncertain or potential threats. Still, anxiety disorders can develop when anxiety or fear responding is excessive or occurs in the absence of true threat, either immediate or future.

Although fear and anxiety both play a role in all anxiety disorder presentations, some argue that anxiety and stress 
disorders can be placed along a fear-anxiety continuum, with PD and phobias classified as fear-based disorders and PTSD and GAD as primarily anxiety-based disorders. ${ }^{5}$ In fact, evidence from rodent models of fear and anxiety suggests that different neural mechanisms may underlie fear and anxiety states. For example, amygdala lesions block fear responding to predictable and identifiable threat but do not affect prolonged anxiety states in response to uncertain future threat. In contrast, lesions of the bed nucleus of the stria terminalis diminish anxiety states while leaving phasic fear responses intact. ${ }^{7}$ A study examining physiological responses to threat in humans demonstrated a gradient of fear reactivity across the anxiety disorder spectrum, with exaggerated fear reactivity in SP, diminished fear reactivity in GAD and PTSD, and intermediate levels of fear reactivity in the other anxiety disorders. ${ }^{8}$ These findings support the notion that fear and anxiety might be somewhat distinct processes, playing different roles across the spectrum of anxiety and stress disorders.

To identify neural circuits underlying fear and anxiety symptomatology, specific experimental paradigms have been developed. ${ }^{9}$ Both rodent and human studies of fear typically employ a fear conditioning paradigm, which pairs an aversive stimulus (eg, electric shock) with a neutral stimulus (eg, a blue light), resulting in the neutral stimulus becoming a signal of imminent threat. This experimental manipulation permits dissociation of brain circuits that imbue "fear" from those involved in the processing of the same or identical stimuli without fear content. A number of key structures have been identified that generate and modulate fear responses to the conditioned threat signal. Specifically, thalamus integrates sensory input from the primary sensory cortices and sends output to the amygdala. The amygdala and dorsal anterior cingulate cortex (dACC) process aversive signals and send output to the hypothalamus, basal ganglia, and brainstem to produce defensive behaviors. ${ }^{10,11}$ The hippocampus is responsible for encoding contextual information associated with the threat cue. ${ }^{11}$ In this way, the hippocampus has been implicated in the extinction of fear, playing a role in down-regulating amygdala response in safe contexts. The medial prefrontal cortex (mPFC) provides topdown regulatory control of fear responding, receiving input from the hippocampus and thalamus and projecting to the amygdala to modulate fear behavior on the basis of complex environmental information. ${ }^{10}$ Overall, these findings support involvement of thalamus, amygdala, dACC, hypothalamus, hippocampus, and $\mathrm{mPFC}$ in fear circuitry. ${ }^{12}$ These regions work in concert to both generate and modulate fear responses to imminent and identifiable threat.
Similarly, both rodent and human studies have been used to identify regions involved in anxiety. Typically, these studies involve unpredictable presentation of an aversive stimulus, leading to the development of vigilance, tension, anticipation, and worry. Many of the same regions making up the fear circuitry also underlie anxiety. Some overlapping regions implicated in both fear and anxiety circuitry include the thalamus, amygdala, and dACC. ${ }^{13}$ In addition, the insula ${ }^{14,15}$ is implicated in vigilance during unpredictable threat, highlighting its role in anxiety circuitry. The bed nucleus of the stria terminalis receives input from the hippocampus, amygdala, and $\mathrm{mPFC}$ and mediates anxiety-related behaviors. ${ }^{16}$ Emotion regulation regions common to both fear and anxiety include the $\mathrm{mPFC}$ and hippocampus. One key difference is the involvement of additional regions in anxiety involved in emotion regulation and attention modulation, including rostral anterior cingulate cortex (rACC) and dorsolateral PFC. . $2,17^{2}$

Three reviews recently summarized structural and functional abnormalities in brain circuitry across the anxiety and stress disorders. ${ }^{13,18,19}$ Functional imaging tasks used to probe neurocircuitry of anxiety disorders mostly include symptom provocation through presentation of disorder-related visual or auditory cues, emotional faces, or fear conditioning. Connectivity analyses examine the co-activation of different brain regions in response to the above-mentioned tasks. The purpose of the current work was to review the recent literature and summarize knowledge gained since 2010. We used PubMed to search the literature published from January 2010 until August 2014, with key terms similar to and including combinations of the following: anxiety, social anxiety disorder, social phobia, panic disorder, specific phobia, posttraumatic stress disorder, generalized anxiety disorder, fMRI (functional magnetic resonance imaging), MRI, PET (positron emission tomography), DTI (diffusion tensor imaging), and neuroimaging. Articles were included if they examined brain structure or function in anxiety on the basis of information presented in the abstract section. On the basis of the articles reviewed, we present a breakdown of the structural and functional abnormalities identified for various anxiety disorders, as well as results pertaining to connectivity between brain regions (see Table 1 for summary of articles reviewed). Our main aims were to synthesize the current literature to identify the neural networks underlying anxiety disorder presentations and to determine whether brain structure, function, and connectivity findings point to similar or independent neural networks across anxiety and stress disorders. We expected to find a consistent pattern of hyperactivation of brain areas underlying fear generation 


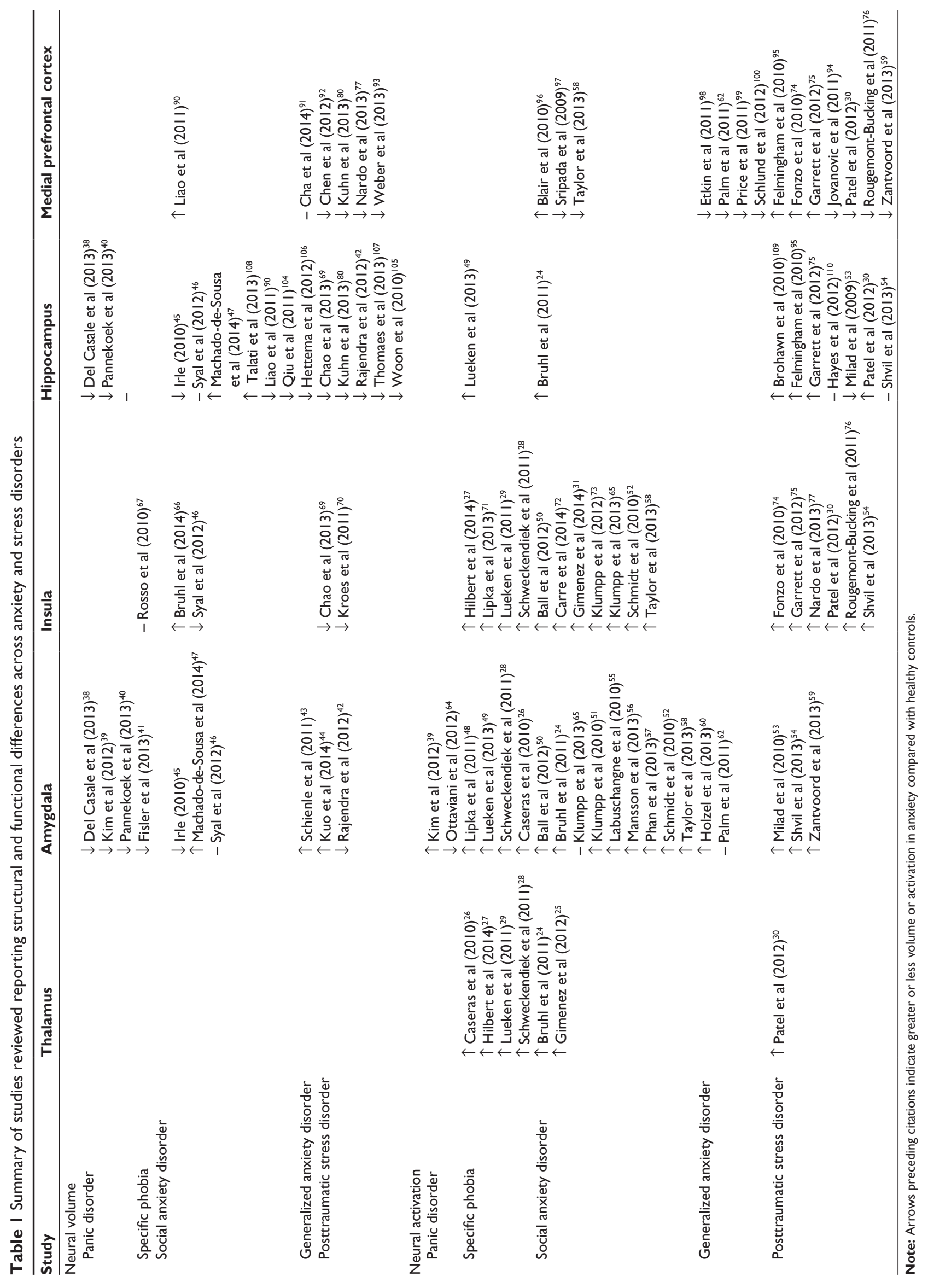


(amygdala, insula, dACC) in more fear-related anxiety disorders (PD, SP) and the same pattern coupled with deficits in emotion modulation areas (mPFC and rACC) in disorders with more prominent anxiety and cognitive components (GAD, PTSD, SAD).

\section{Sensory processing regions}

According to rodent models and human imaging studies, a number of regions responsible for taking in and processing sensory information, such as the occipital cortex, fusiform gyrus, and thalamus, have been implicated in anxiety disorder neurocircuitry. ${ }^{11,20}$ The main finding reported is increased activation in these regions in response to threatening stimuli in anxious patients compared to healthy controls.

\section{Occipital cortex and fusiform gyrus}

The occipital cortex, a region responsible for processing visual stimuli, is more active in response to threatening images in anxious patients, particularly those with SAD, compared with controls. In patients with $\mathrm{SAD}$, greater activation in occipitotemporal regions predicted $\mathrm{D}$-cycloserine treatment response, with greater pretreatment activation associated with a greater decrease in symptoms posttreatment. ${ }^{21}$ The fusiform gyrus contains neurons specific to face perception, known as the fusiform face area, ${ }^{22}$ which is more active in response to threat faces in SAD compared with healthy controls. ${ }^{23}$

\section{Thalamus}

The thalamus is implicated in sensory integration, and functional imaging studies revealed increased activation in the thalamus in response to phobic-related and threat stimuli in patients with $\mathrm{SAD},{ }^{24,25}$ blood-injection-injury phobia, ${ }^{26}$ dental phobia, ${ }^{27}$ spider phobia, ${ }^{28}$ snake phobia, ${ }^{29}$ and PTSD. ${ }^{30}$ Contrary to these findings, one study reported that dental phobics did not demonstrate increased activation in thalamus to phobic images. ${ }^{29}$ Activation in the thalamus was correlated with the degree of anxiety and disgust in blood-injury-injection phobia, ${ }^{26}$ as well as autonomic arousal in snake phobia, but not in dental phobia. ${ }^{29}$ Treatment with paroxetine reduced activation in thalamus compared with placebo treatment in response to a recorded performance task in SAD. ${ }^{31}$

\section{Emotion generating/processing regions}

Ample evidence from basic and human imaging studies suggests that regions such as the striatum, ${ }^{32}$ amygdala, ${ }^{10,33,34}$ insula, ${ }^{11,14,15}$ and $\mathrm{dACC}{ }^{35}$ play a large role in identifying fear stimuli and generating fear responses. These areas often have structural abnormalities and are hyperactive in anxiety compared to controls. Extensive prior evidence ${ }^{13}$ suggests hyperactivation in the amygdala across all anxiety disorders. Hyperactivation was also reported in insula in SP, SAD, PTSD, and GAD, whereas activation differences were less consistent in PD. Hyperactivation in dACC was reported in SP and PTSD, with mixed findings in PD and SAD and limited evidence in GAD.

\section{Striatum}

Less activation in ventral striatum has been reported in SAD while anticipating giving a speech, with greater levels of anticipatory anxiety predicting less activation. ${ }^{36}$ Although striatal activation is typically modulated in response to social cooperation, this was not observed in SAD, suggesting abnormalities in reward circuitry related to the initiation and maintenance of social relationships. ${ }^{37}$

\section{Amygdala}

Studies examining structural differences in the amygdala in anxiety patients compared with healthy controls find decreased amygdala volume and density in $\mathrm{PD},{ }^{38-40} \mathrm{SP},{ }^{41}$ and PTSD, ${ }^{42}$ with symptom severity predictive of smaller amygdala volume. ${ }^{41}$ In contrast, some studies reported larger amygdala volume in anxiety, specifically $\mathrm{GAD}^{43}$ and PTSD. ${ }^{44}$ The picture for SAD is more complex, with reports of reduced amygdala volume, ${ }^{45}$ no differences in amygdala volume, ${ }^{46}$ and larger amygdala volume ${ }^{47}$ in SAD compared with controls.

Recent functional imaging studies report amygdala hyperactivation in response to threatening stimuli in $\mathrm{PD},{ }^{39}$ SP, ${ }^{28,48,49}$ SAD,${ }^{24,36,50-52}$ and PTSD ${ }^{53,54}$ compared with healthy controls. The degree of amygdala activation was positively correlated with symptom severity in $\mathrm{SP}^{48}$ and SAD. ${ }^{50}$ Moreover, treatment with medication and psychotherapy often results in decreased amygdala hyperactivation to threat from pre- to posttreatment in SP, ${ }^{48} \mathrm{SAD},{ }^{55-58}$ and PTSD. ${ }^{59}$ Patterns of amygdala activation in GAD are more complex, with studies reporting increased ${ }^{60,61}$ or no difference ${ }^{62}$ in activation in GAD compared with healthy controls. Null and opposing findings suggesting no difference or decreased amygdala activation in anxiety compared with controls have also been reported in $\mathrm{PD},{ }^{63,64} \mathrm{SP},{ }^{49}$ and $\mathrm{SAD},{ }^{65}$ with some evidence that medication treatment (paroxetine) increases amygdala activation in SAD compared with healthy controls. ${ }^{31}$ 


\section{Insula}

Structural imaging results revealed conflicting reports of increased volume in $\mathrm{SAD},{ }^{66}$ no difference in $\mathrm{SP},{ }^{67}$ and decreased volume in $\mathrm{SAD}^{46}$ and PTSD ${ }^{68-70}$ compared with controls.

Recent functional imaging studies revealed insula hyperactivation to threat in patients with $\mathrm{SP},{ }^{27-29,71} \mathrm{SAD},{ }^{36,50,52,65,72,73}$ and PTSD ${ }^{30,54,74-77}$ compared with controls. The degree of insula hyperactivation was positively correlated with symptom severity in SAD. ${ }^{72}$ Both medication and psychotherapy have been shown to decrease insula hyperactivation from preto posttreatment in SAD. ${ }^{31,58,78}$ In contrast, one study found that patients with dental phobia did not show hyperactivation in insula during exposure to phobic stimuli compared with healthy controls. ${ }^{29}$

\section{Dorsal anterior cingulate cortex}

Literature suggests decreased dACC gray matter and white matter volume in $\mathrm{PD},{ }^{40} \mathrm{SP},{ }^{79}$ and $\mathrm{PTSD}{ }^{80-82}$ compared with healthy controls. In contrast, some studies have reported increased volume in $\mathrm{dACC}$ in $\mathrm{SAD}^{66}$ and GAD. ${ }^{43}$

Functional imaging studies reported dACC hyperactivation to threat in SP, ${ }^{26-29,71} \mathrm{SAD},{ }^{24,50,83}$ and PTSD. ${ }^{30,84-86}$ Increased $\mathrm{dACC}$ activation was correlated with greater autonomic arousal ${ }^{29}$ and subjective anxiety levels ${ }^{26,71}$ in SP. Cognitive behavioral therapy was found to decrease ACC activation in SP, ${ }^{71}$ and pretreatment ACC activation predicted positive treatment response in patients with GAD. ${ }^{61}$ Despite relatively consistent findings of hyperactivation in dACC, some idiosyncrasies exist in the literature. In contrast to other types of phobia, patients with dental phobia ${ }^{26}$ and blood-injection-injury phobia ${ }^{29}$ did not exhibit increased ACC activation to phobic stimuli relative to healthy controls, suggesting dACC may not be involved in threat processing in some anxiety symptom presentations. Moreover, one study reported that $\mathrm{dACC}$ was less active to threat in SAD compared with healthy controls. ${ }^{87}$

\section{Emotion modulation regions}

Regions involved in regulating threat responding are particularly important in anxiety, as they can decrease activation in threat-processing regions such as the amygdala, insula, and dACC. These have been identified using basic science models and human imaging studies. The mPFC, hippocampus, dorsolateral prefrontal cortex (dlPFC), and rACC have been implicated in modulating fear responding. Although the $\mathrm{mPFC}^{10,33,34,88}$ and $\mathrm{rACC}^{89}$ are primarily involved in modulating emotion, the $\mathrm{dlPFC}^{89}$ has been implicated in both emotion modulation and attention control. The hippocampus is primarily involved in encoding contextual information and modulating fear responding within the context of threat and safety signals. ${ }^{11}$ As such, these regions underlie different functions that may work in concert to modulate threat response. Evidence reported before the scope of the current review $^{13}$ suggests hyperactivation in the hippocampus in PD and PTSD but little evidence for hippocampus involvement in other anxiety disorders. Hypoactivation in the mPFC has been reported in PTSD and GAD, with less consistent results seen in PD, SP, and SAD. Evidence for dlPFC and rACC is less consistent and less studied, with both hyper- and hypoactivation reported in PD, SP, SAD, and PTSD.

\section{Medial prefrontal cortex}

Structural imaging studies report differences in $\mathrm{MPFC}$, with increased volume in SAD, ${ }^{90}$ decreased volume in PTSD, ${ }^{77,80}$ and no difference in GAD, ${ }^{91}$ with decreased volume associated with greater symptom severity in PTSD. ${ }^{92,93}$

Functional imaging studies, including two recent metaanalyses and a literature review, ${ }^{30,76,94}$ primarily report decreased mPFC activation in PTSD compared with healthy controls. Some other studies of PTSD patients reported increased mPFC activation in response to fearful faces. ${ }^{74,75,95}$ Similar findings are reported in SAD, with both increased ${ }^{96}$ and decreased ${ }^{97}$ mPFC activation in response to threat and social tasks. Results are somewhat more consistent in GAD, with the majority reporting decreased mPFC activation. ${ }^{62,98-100}$ It has been suggested that hyperactivation and hypoactivation in mPFC may be associated with different symptom profiles. ${ }^{101}$ It is also possible that although hypoactivation indicates a deficit in emotion regulation, hyperactivation indicates an overcompensatory response in an effort to decrease excessive fear responding. Regardless of these discrepancies, the treatment literature is quite consistent, suggesting that both pharmacotherapy and psychotherapy produce increases in $\mathrm{mPFC}$ activation in $\mathrm{SAD}^{57,58,102,103}$ and PTSD, ${ }^{59}$ which is related to symptom improvements.

\section{Hippocampus}

Although the hippocampus is often considered part of the limbic system responsible for fear generation, the majority of evidence in rodent and human models examining hippocampal function suggests its primary role is in context learning and fear modulation in the presence of safety and threat contexts. ${ }^{11}$ Structural imaging studies of the hippocampus suggest decreased volume and density in $\mathrm{PD},{ }^{38,40}$ SAD, ${ }^{45,104,90}$, GAD, ${ }^{106}$ and PTSD. ${ }^{42,69,80,105}$ There is also evidence for increased hippocampal volume after treatment with 
selective serotonin reuptake inhibitors in PTSD. ${ }^{107}$ However, findings are somewhat mixed, with studies also reporting no differences $^{46}$ and larger ${ }^{47,108}$ hippocampal volume in SAD compared with healthy controls.

Functional imaging studies report increased hippocampal activation to threat in $\mathrm{SP},{ }^{49} \mathrm{SAD},{ }^{24}$ and $\mathrm{PTSD}^{30,75,95}$ compared with healthy controls. Increased hippocampal activation was related to defensive reactivity in response to threat stimuli in SP. ${ }^{49}$ Differences in hippocampus activation in PTSD during memory tasks ${ }^{53,109,110}$ and emotional activation tasks ${ }^{54}$ have been less consistent, with both increased and decreased activation reported.

\section{Other modulatory regions}

Evidence exists suggesting that other regions, including the dlPFC and subgenual/rACC, play a role in emotion modulation. ${ }^{89}$ Structural imaging studies report increased dlPFC volume and thickness in SP, ${ }^{111}$ with larger volume predicting more severe symptoms and arousal levels.

Functional imaging studies report dlPFC hyperactivation to threat in $\mathrm{SP}^{111}$ compared with controls. In addition, dIPFC mediated the influence of SAD on laughter perception, which was related to symptom severity. ${ }^{112}$ Hypoactivation in $\mathrm{rACC}$ has been reported in SAD, ${ }^{65}$ PTSD, ${ }^{94,113}$ and GAD. ${ }^{62,100,114}$ However, treatment with computerized attention training ${ }^{58}$ and paroxetine ${ }^{31}$ reduced activation in subgenual ACC in SAD.

\section{Connectivity between regions}

More recent studies have begun to focus on connectivity between brain regions in anxiety patients. Results overwhelmingly suggest decreased connectivity between emotion processing (amygdala, insula) and emotion modulation (mPFC, rACC) regions. This finding is consistent both during rest and while performing a variety of cognitive and emotional tasks and is often interpreted as a deficit in regulating fear responding. Studies examining the structural connectivity between medial-frontal and basal-limbic areas, including the amygdala, measured via volume of the uncinated fasciculus, revealed smaller volume in the left uncinated fasciculus in patients with SAD than in controls. This finding suggests communication deficits between emotion-generating and emotion-modulation regions in SAD. ${ }^{115}$

Functional connectivity analyses reveal less connectivity between amygdala and $\mathrm{mPFC}$ in $\mathrm{SAD},{ }^{116} \mathrm{PTSD},{ }^{94}$ and GAD. ${ }^{117-120}$ There is also evidence for reduced connectivity between the amygdala and ventrolateral prefrontal cortex (vlPFC) in GAD, ${ }^{60,121}$ and the amygdala and subgenual/rACC in $\mathrm{SAD}^{122}$ and GAD ${ }^{98,114,117}$ Connectivity improves after treatment for $\mathrm{GAD}^{60,123}$ and SAD. ${ }^{116}$ Some conflicting findings do exist, however, with reports of increased connectivity between dorsomedial prefrontal cortex (dmPFC) and amygdala ${ }^{103}$ and between $\mathrm{mPFC}$ and amygdala ${ }^{124}$ in patients with SAD compared with controls.

Differences in connectivity within emotion-processing regions are also reported in anxiety compared with controls in a small number of studies, with the majority reporting decreased connectivity. Specifically, reports show decreased connectivity between insula and $\mathrm{dACC}$ in $\mathrm{SAD}^{73}$ and between amygdala and insula in GAD. ${ }^{120,125}$ However, increased connectivity between amygdala and insula was reported in PTSD. ${ }^{126}$

More global differences in connectivity have been observed in SAD, with differences in gray matter volume across the whole brain ${ }^{127}$ and deficits in global brain networks including the default mode network and the central-executive network compared with healthy controls. ${ }^{104}$ Deficits in connectivity were also observed in and between regions involved in general arousal and attention. ${ }^{25}$

\section{Discussion}

This review of recent literature suggests the presence of both common and distinct neural circuits involved in anxiety disorders. It partially supports the idea of a spectrum, with more cue-related and fear-based disorders (PD, SP) on one end and anxiety-based disorders (GAD, SAD, and PTSD) on the other. In general, all disorders involved deficits in both emotion-generating regions and modulatory regions, suggesting fear and anxiety both play key roles across the anxiety spectrum. What differentiates disorders appears to be the degree of dispersion of functional changes across the brain. Although PD and SP are characterized by deficits primarily in emotion-generating regions, SAD is characterized by deficits in a number of additional regions involved in sensory processing and attentional control, GAD findings are the least consistent in the emotion-generating circuit and most consistent in the frontal emotion regulatory circuit, while PTSD had a relatively consistent pattern in both circuits.

\section{Common neurocircuitry}

Changes in amygdala, ACC, and hippocampus are implicated across all anxiety disorders by multiple studies. Structural imaging studies report overall mixed results, with reports of both increased and decreased regional volumes in anxiety patients. Functional neuroimaging findings are more consistent, however, with the majority of studies reporting 
hyperactive amygdala response to threat across anxiety disorders. The hippocampus is reported as hyperactive across multiple anxiety disorders; however, what aspect of hippocampal function this hyperactivity reflects is less clear. Although hyperactivation in hippocampus seems to parallel hyperactivation observed in amygdala, the prototypic emotion-generating structure, basic rodent and human models of anxiety suggest the hippocampus is primarily a regulatory region. It is possible that the hippocampus is involved in multiple processes related to both fear generation and modulation, consistent with the view of "neural reuse," arguing that brain regions can be used for multiple functions. ${ }^{128}$

ACC activation varied as a function of subregions: dorsal, rostral, and subgenual. In general, it seems that across anxiety disorders, activation in $\mathrm{dACC}$ is increased in response to threat, whereas subgenual/rACC activation patterns are less consistent, although hypoactivation was the most common finding in SAD, PTSD, and GAD. Although differences in activation patterns may depend on the task and stimuli used, the different functions of ACC subregions and the connectivity of these subregions to other brain structures likely contribute to these findings. Specifically, although dACC appears to be involved in threat processing and fear generation, subgenual/rACC is primarily involved in emotion modulation.

Connectivity analysis suggests a common mechanism for relationships between PFC and limbic structures across anxiety disorders. Specifically, decreased connectivity between emotion-generating areas (amygdala, insula) and cortical regulatory regions ( $\mathrm{mPFC}, \mathrm{rACC}$ ) have been consistently found across anxiety disorders. Connectivity was inversely related to symptom severity, and in a number of studies employing pre-post design, it increased after anxiety treatment. Overall, these findings point to a potential deficit in fear regulation circuitry in anxiety consisting of hyperactivation of emotion-generating regions coupled with dysfunction in emotion-regulation regions. This suggests the possibility of a shared network underlying the spectrum of anxiety and stress disorders.

\section{Distinct neurocircuits}

Activation patterns in amygdala and hippocampus were less consistent in GAD. In contrast, hypoactivation in the subgenual/rACC and $\mathrm{mPFC}$ are more consistent in this disorder. The primary clinical presentation of GAD is constant and unfocused worries about ambiguous and potentially negative future outcomes, rather than a focus on a specific threat. This cognitive component of GAD, including distorted beliefs and ruminations, may explain consistent deficits in emotion regulatory areas. In other words, GAD may be more "anxiety related" than "fear related," where impaired function of the prefrontal cortical areas has a "permissive" role in increased anxiety. In SAD, the changes in neural activation patterns also appeared more distributed (beyond the set of limbic and prefrontal regions discussed earlier) compared with other anxiety disorders. SAD studies report increased activation in the fusiform face area and occipital cortex, indicating enhanced stimulus processing associated with anxiety. The increased hypersensitivity to social cues and self-reference typical in SAD could account for differences in these brain regions that may be less relevant in other anxiety disorders.

More consistent reports of hypoactivation in $\mathrm{mPFC}$ regions in disorders that are closer to the anxiety than to the fear end of the spectrum (ie, GAD, SAD, and PTSD) may relate to the cognitive processes and diffuse anxiety states associated with these disorders, leading to more anxietylike cognitions and ruminations. Decreased activation in mPFC was observed consistently in PTSD and GAD, with mixed findings in SAD. In contrast, dlPFC hyperactivation to threat was observed in SP, and this region also appears to regulate responses to an ambiguous cue (laughter) in SAD. Hypoactivation in emotion regulation regions such as $\mathrm{mPFC}$ is typically interpreted as a deficit in ability to appropriately inhibit threat responding in anxiety. However, a number of studies also report hyperactivation in $\mathrm{mPFC}$ in anxiety. One possible explanation for hyperactivation is that these regions are overcompensating in an effort to down-regulate activity in amygdala and other emotion-generating regions. We may also be observing a time delay with respect to when these regulatory regions are coming online in anxious compared with healthy controls, resulting in activation differences. In other words, although the main changes observed in fearrelated anxiety disorders such as SP or PD may reflect hyperreactivity of the emotion-generating system to threat/feared cues, distorted anxiety-related cognitions may play a larger role in GAD, SAD, and PTSD.

The existing literature examining structural and functional deficits in fear and anxiety circuitry is quickly growing, and just in the last 5 years, a considerable amount of knowledge regarding common and distinct patterns of neural function across the anxiety and stress disorder spectrum has been gained. However, much remains to be learned about specific differences in brain function across both emotion-generating and emotion-regulating regions. Future studies should focus on striving for consistency in paradigms used and patient 
populations studied to improve our ability to compare and contrast findings across experiments. It is also imperative to continue investigating connectivity between brain regions across the spectrum of anxiety and stress disorders to fully identify neural circuitry underlying symptom presentations, rather than simple activation patterns. It is clear that threat processing and emotion regulation circuits interact with one another, making more global and interactive analyses key in understanding these complex processes. Additional treatment studies are also indicated in an effort to better understand how pharmacologic and psychotherapeutic approaches can be used and modified to best target and correct aberrant brain function underlying anxiety.

\section{Disclosure}

The authors report no conflicts of interest in this work.

\section{References}

1. Kessler RC, Berglund P, Demler O, Jin R, Merikangas KR, Walters EE. Lifetime prevalence and age-of-onset distributions of DSM-IV disorders in the National Comorbidity Survey Replication. Arch Gen Psychiatry. 2005;62(6):593-602.

2. Greenberg PE, Sisitsky T, Kessler RC, et al. The economic burden of anxiety disorders in the 1990s. J Clin Psychiatry. 1999;60(7): 427-435.

3. Wittchen H-U. Generalized anxiety disorder: prevalence, burden, and cost to society. Depress Anxiety. 2002;16(4):162-171.

4. Kessler RC. The impairments caused by social phobia in the general population: implications for intervention. Acta Psychiatr Scand Suppl. 2003;108(417):19-27.

5. American Psychiatric Association. The Diagnostic and Statistical Manual of Mental Disorders. 5th ed. Arlington, VA: American Psychiatric Association; 2013.

6. Barlow DH. Unraveling the mysteries of anxiety and its disorders from the perspective of emotion theory. Am Psychol. 2000;55(11): 1247-1263.

7. Davis M, Walker DL, Miles L, Grillon C. Phasic vs sustained fear in rats and humans: role of the extended amygdala in fear vs anxiety. Neuropsychopharmacology. 2010;35(1):105-135.

8. McTeague LM, Lang PJ. The anxiety spectrum and the reflex physiology of defense: from circumscribed fear to broad distress. Depress Anxiety. 2012;29(4):264-281.

9. Schmitz A, Grillon C. Assessing fear and anxiety in humans using the threat of predictable and unpredictable aversive events (the NPU-threat test). Nat Protoc. 2012;7(3):527-532.

10. Lee S, Kim S-J, Kwon O-B, Lee JH, Kim J-H. Inhibitory networks of the amygdala for emotional memory. Front Neural Circuits. 2013; 7:129.

11. Maren S. Neurobiology of Pavlovian fear conditioning. Annu Rev Neurosci. 2001;24(1):897-931.

12. Kumar V, Bhat ZA, Kumar D. Animal models of anxiety: a comprehensive review. J Pharmacol Toxicol Methods. 2013;68(2):175-183.

13. Shin LM, Liberzon I. The neurocircuitry of fear, stress, and anxiety disorders. Neuropsychopharmacology. 2010;35(1):169-191.

14. Stein MB, Simmons AN, Feinstein JS, Paulus MP. Increased amygdala and insula activation during emotion processing in anxiety-prone subjects. Am J Psychiatry. 2007;164(2):318-327.

15. Shankman SA, Gorka SM, Nelson BD, FitzgeraldDA, Phan KL, O’Daly O. Anterior insula responds to temporally unpredictable aversiveness: an fMRI study. Neuroreport. 2014;25(8):596-600.
16. Taugher RJ, Lu Y, Wang Y, et al. The bed nucleus of the stria terminalis is critical for anxiety-related behavior evoked by $\mathrm{CO} 2$ and acidosis. J Neurosci. 2014;34(31):10247-10255.

17. Kollack-Walker S, Watson SJ, Akil H. Social stress in hamsters: defeat activates specific neurocircuits within the brain. J Neurosci. 1997;17(22):8842-8855. Available from: http://www.ncbi.nlm.nih. gov/pubmed/9348352. Accessed October 31, 2014.

18. Damsa C, Kosel M, Moussally J. Current status of brain imaging in anxiety disorders. Curr Opin Psychiatry. 2009;22(1):96-110.

19. Engel K, Bandelow B, Gruber O, Wedekind D. Neuroimaging in anxiety disorders. J Neural Transm. 2009;116(6):703-716.

20. Lang PJ, Bradley MM, Cuthbert BN. Emotion, motivation, and anxiety: brain mechanisms and psychophysiology. Biol Psychiatry. 1998; 44(12):1248-1263.

21. Doehrmann O, Ghosh SS, Polli FE, et al. Predicting treatment response in social anxiety disorder from functional magnetic resonance imaging. JAMA Psychiatry. 2013;70(1):87-97.

22. Kanwisher N, McDermott J, Chun MM. The fusiform face area: a module in human extrastriate cortex specialized for face perception. JNeurosci. 1997;17(11):4302-4311. Available from: http://www.ncbi. nlm.nih.gov/pubmed/9151747. Accessed October 31, 2014.

23. Frick A, Howner K, Fischer H, Kristiansson M, Furmark T. Altered fusiform connectivity during processing of fearful faces in social anxiety disorder. Transl Psychiatry. 2013;3(10):e312.

24. Brühl AB, Rufer M, Delsignore A, Kaffenberger T, Jäncke L, Herwig U. Neural correlates of altered general emotion processing in social anxiety disorder. Brain Res. 2011;1378:72-83.

25. Giménez M, Pujol J, Ortiz H, et al. Altered brain functional connectivity in relation to perception of scrutiny in social anxiety disorder. Psychiatry Res. 2012;202(3):214-223.

26. Caseras X, Giampietro V, Lamas A, et al. The functional neuroanatomy of blood-injection-injury phobia: a comparison with spider phobics and healthy controls. Psychol Med. 2010;40(1):125-134.

27. Hilbert K, Evens R, Maslowski NI, Wittchen HU, Lueken U. Fear processing in dental phobia during crossmodal symptom provocation: an fMRI study. Biomed Res Int. 2014;2014:196353.

28. Schweckendiek J, Klucken T, Merz CJ, et al. Weaving the (neuronal) web: fear learning in spider phobia. Neuroimage. 2011;54(1):681-688.

29. Lueken U, Kruschwitz JD, Muehlhan M, Siegert J, Hoyer J, Wittchen H-U. How specific is specific phobia? Different neural response patterns in two subtypes of specific phobia. Neuroimage. 2011;56(1):363-372.

30. Patel R, Spreng RN, Shin LM, Girard TA. Neurocircuitry models of posttraumatic stress disorder and beyond: a meta-analysis of functional neuroimaging studies. Neurosci Biobehav Rev. 2012;36(9): 2130-2142.

31. Giménez M, Ortiz H, Soriano-Mas C, et al. Functional effects of chronic paroxetine versus placebo on the fear, stress and anxiety brain circuit in Social Anxiety Disorder: initial validation of an imaging protocol for drug discovery. Eur Neuropsychopharmacol. 2014;24(1):105-116.

32. Ferry AT, Ongür D, An X, Price JL. Prefrontal cortical projections to the striatum in macaque monkeys: evidence for an organization related to prefrontal networks. J Comp Neurol. 2000;425(3):447-470. Available from: http://www.ncbi.nlm.nih.gov/pubmed/10972944. Accessed October 31, 2014.

33. Herry C, Ferraguti F, Singewald N, Letzkus JJ, Ehrlich I, Lüthi A. Neuronal circuits of fear extinction. Eur J Neurosci. 2010;31(4):599-612.

34. Marek R, Strobel C, Bredy TW, Sah P. The amygdala and medial prefrontal cortex: partners in the fear circuit. J Physiol. 2013;591(Pt 10): 2381-2391.

35. Whalen PJ, Bush G, McNally RJ, et al. The emotional counting Stroop paradigm: a functional magnetic resonance imaging probe of the anterior cingulate affective division. Biol Psychiatry. 1998;44(12):1219-1228.

36. Boehme S, Ritter V, Tefikow S, et al. Brain activation during anticipatory anxiety in social anxiety disorder. Soc Cogn Affect Neurosci. 2014;9(9):1413-1418.

37. Sripada C, Angstadt M, Liberzon I, McCabe K, Phan KL. Aberrant reward center response to partner reputation during a social exchange game in generalized social phobia. Depress Anxiety. 2013;30(4):353-361. 
38. Del Casale A, Serata D, Rapinesi C, et al. Structural neuroimaging in patients with panic disorder: findings and limitations of recent studies. Psychiatr Danub. 2013;25(2):108-114. Available from: http://www. ncbi.nlm.nih.gov/pubmed/23793273. Accessed October 31, 2014.

39. Kim JE, Dager SR, Lyoo IK. The role of the amygdala in the pathophysiology of panic disorder: evidence from neuroimaging studies. Biol Mood Anxiety Disord. 2012;2(1):20.

40. Pannekoek JN, van der Werff SJ, Stein DJ, van der Wee NJ. Advances in the neuroimaging of panic disorder. Hum Psychopharmacol. 2013; 28(6):608-611.

41. Fisler MS, Federspiel A, Horn H, et al. Spider phobia is associated with decreased left amygdala volume: a cross-sectional study. $B M C$ Psychiatry. 2013;13(1):70.

42. Rajendra A Morey, Andrea L Gold, Kevin S LaBar, et al. Amygdala volume changes with posttraumatic stress disorder in a large casecontrolled veteran group. Arch Gen Psychiatry. 2012;69(11): 1169-1178.

43. Schienle A, Ebner F, Schäfer A. Localized gray matter volume abnormalities in generalized anxiety disorder. Eur Arch Psychiatry Clin Neurosci. 2011;261(4):303-307.

44. Kuo JR, Kaloupek DG, Woodward SH. Amygdala volume in combatexposed veterans with and without posttraumatic stress disorder: a crosssectional study. Arch Gen Psychiatry. 2012;69(10):1080-1086.

45. Irle E, Ruhleder M, Lange $C$, et al. Reduced amygdalar and hippocampal size in adults with generalized social phobia. J Psychiatry Neurosci. 2010;35(2):126-131.

46. Syal S, Hattingh CJ, Fouché J-P, et al. Grey matter abnormalities in social anxiety disorder: a pilot study. Metab Brain Dis. 2012;27(3): 299-309.

47. Machado-de-Sousa JP, Osório FL, Jackowski AP, et al. Increased amygdalar and hippocampal volumes in young adults with social anxiety. PLoS ONE. 2014;9(2):e88523.

48. Lipka J, Miltner WHR, Straube T. Vigilance for threat interacts with amygdala responses to subliminal threat cues in specific phobia. Biol Psychiatry. 2011;70(5):472-478.

49. Lueken U, Hilbert K, Stolyar V, Maslowski NI, Beesdo-Baum K, Wittchen HU. Neural substrates of defensive reactivity in two subtypes of specific phobia. Soc Cogn Affect Neurosci. Epub 2013 Nov 14.

50. Ball TM, Sullivan S, Flagan T, et al. Selective effects of social anxiety, anxiety sensitivity, and negative affectivity on the neural bases of emotional face processing. Neuroimage. 2012;59(2):1879-1887.

51. Klumpp H, Angstadt M, Nathan PJ, Phan KL. Amygdala reactivity to faces at varying intensities of threat in generalized social phobia: an event-related functional MRI study. Psychiatry Res. 2010;183(2): 167-169.

52. Schmidt S, Mohr A, Miltner WHR, Straube T. Task-dependent neural correlates of the processing of verbal threat-related stimuli in social phobia. Biol Psychol. 2010;84(2):304-312.

53. Milad MR, Pitman RK, Ellis CB, et al. Neurobiological basis of failure to recall extinction memory in posttraumatic stress disorder. Biol Psychiatry. 2009;66(12):1075-1082.

54. Shvil E, Rusch HL, Sullivan GM, Neria Y. Neural, psychophysiological, and behavioral markers of fear processing in PTSD: a review of the literature. Curr Psychiatry Rep. 2013;15(5):358-374.

55. Labuschagne I, Phan KL, Wood A, et al. Oxytocin attenuates amygdala reactivity to fear in generalized social anxiety disorder. Neuropsychopharmacology. 2010;35(12):2403-2413.

56. Månsson KNT, Carlbring P, Frick A, et al. Altered neural correlates of affective processing after internet-delivered cognitive behavior therapy for social anxiety disorder. Psychiatry Res. 2013;214(3): 229-237.

57. Phan KL, Coccaro EF, Angstadt M, et al. Corticolimbic brain reactivity to social signals of threat before and after sertraline treatment in generalized social phobia. Biol Psychiatry. 2013;73(4):329-336.

58. Taylor CT, Aupperle RL, Flagan T, et al. Neural correlates of a computerized attention modification program in anxious subjects. Soc Cogn Affect Neurosci. 2014;9(9):1379-1387.
59. Zantvoord JB, Diehle J, Lindauer RJL. Using neurobiological measures to predict and assess treatment outcome of psychotherapy in posttraumatic stress disorder: systematic review. Psychother Psychosom. 2013; 82(3):142-151.

60. Hölzel BK, Hoge EA, Greve DN, et al. Neural mechanisms of symptom improvements in generalized anxiety disorder following mindfulness training. Neuroimage Clin. 2013;2:448-458.

61. Nitschke JB, Sarinopoulos I, Oathes DJ, et al. Anticipatory activation in the amygdala and anterior cingulate in generalized anxiety disorder and prediction of treatment response. Am J Psychiatry. 2009;166(3):302-310.

62. Palm ME, Elliott R, McKie S, Deakin JFW, Anderson IM. Attenuated responses to emotional expressions in women with generalized anxiety disorder. Psychol Med. 2011;41(5):1009-1018.

63. Boutros NN, Galloway MP, Ghosh S, Gjini K, Bowyer SM. Abnormal coherence imaging in panic disorder: a magnetoencephalography investigation. Neuroreport. 2013;24(9):487-491.

64. Ottaviani C, Cevolani D, Nucifora V, et al. Amygdala responses to masked and low spatial frequency fearful faces: a preliminary fMRI study in panic disorder. Psychiatry Res. 2012;203(2-3):159-165.

65. Klumpp H, Post D, Angstadt M, Fitzgerald DA, Phan KL. Anterior cingulate cortex and insula response during indirect and direct processing of emotional faces in generalized social anxiety disorder. Biol Mood Anxiety Disord. 2013;3(1):7.

66. Brühl AB, Hänggi J, Baur V, et al. Increased cortical thickness in a frontoparietal network in social anxiety disorder. Hum Brain Mapp. 2014;35(7):2966-2977.

67. Rosso IM, Makris N, Britton JC, et al. Anxiety sensitivity correlates with two indices of right anterior insula structure in specific animal phobia. Depress Anxiety. 2010;27(12):1104-1110.

68. Ahmed F, Spottiswoode BS, Carey PD, Stein DJ, Seedat S. Relationship between neurocognition and regional brain volumes in traumatized adolescents with and without posttraumatic stress disorder. Neuropsychobiology. 2012;66(3):174-184.

69. Chao L, Weiner M, Neylan T. Regional cerebral volumes in veterans with current versus remitted posttraumatic stress disorder. Psychiatry Res. 2013;213(3):193-201

70. Kroes MCW, Whalley MG, Rugg MD, Brewin CR. Association between flashbacks and structural brain abnormalities in posttraumatic stress disorder. Eur Psychiatry. 2011;26(8):525-531.

71. Lipka J, Hoffmann M, Miltner WH, Straube T. Effects of CognitiveBehavioral Therapy on Brain Responses to Subliminal and Supraliminal Threat and Their Functional Significance in Specific Phobia. Biol Psychiatry. Epub 2013 Nov 14.

72. Carré A, Gierski F, Lemogne C, et al. Linear association between social anxiety symptoms and neural activations to angry faces: from subclinical to clinical levels. Soc Cogn Affect Neurosci. 2014;9(6):880-886.

73. Klumpp H, Angstadt M, Phan KL. Insula reactivity and connectivity to anterior cingulate cortex when processing threat in generalized social anxiety disorder. Biol Psychol. 2012;89(1):273-276.

74. Fonzo GA, Simmons AN, Thorp SR, Norman SB, Paulus MP, Stein MB. Exaggerated and disconnected insular-amygdalar blood oxygenation level-dependent response to threat-related emotional faces in women with intimate-partner violence posttraumatic stress disorder. Biol Psychiatry. 2010;68(5):433-441

75. Garrett AS, Carrion V, Kletter H, Karchemskiy A, Weems CF, Reiss A. Brain activation to facial expressions in youth with PTSD symptoms. Depress Anxiety. 2012;29(5):449-459.

76. Rougemont-Bücking A, Linnman C, Zeffiro TA, et al. Altered processing of contextual information during fear extinction in PTSD: an fMRI study. CNS Neurosci Ther. 2011;17(4):227-236.

77. Nardo D, Högberg G, Lanius RA, et al. Gray matter volume alterations related to trait dissociation in PTSD and traumatized controls. Acta Psychiatr Scand. 2013;128(3):222-233.

78. Klumpp H, Fitzgerald DA, Phan KL. Neural predictors and mechanisms of cognitive behavioral therapy on threat processing in social anxiety disorder. Prog Neuropsychopharmacol Biol Psychiatry. 2013;45: 83-91. 
79. Linares IMP, Jackowski AP, Trzesniak CM, et al. Cortical thinning of the right anterior cingulate cortex in spider phobia: a magnetic resonance imaging and spectroscopy study. Brain Res. 2014;1576:35-42.

80. Kühn S, Gallinat J. Gray matter correlates of posttraumatic stress disorder: a quantitative meta-analysis. Biol Psychiatry. 2013;73(1): 70-74.

81. Schuff N, Zhang Y, Zhan W, et al. Patterns of altered cortical perfusion and diminished subcortical integrity in posttraumatic stress disorder: an MRI study. Neuroimage. 2011;54 Suppl 1:S62-S68.

82. Zhang J, Tan Q, Yin H, et al. Decreased gray matter volume in the left hippocampus and bilateral calcarine cortex in coal mine flood disaster survivors with recent onset PTSD. Psychiatry Res. 2011;192(2): 84-90.

83. Duval ER, Hale LR, Liberzon I, et al. Anterior cingulate cortex involvement in subclinical social anxiety. Psychiatry Res. 2013;214(3): 459-461.

84. Ramage AE, Laird AR, Eickhoff SB, et al. A coordinate-based metaanalytic model of trauma processing in posttraumatic stress disorder. Hum Brain Mapp. 2013;34(12):3392-3399.

85. Sartory G, Cwik J, Knuppertz H, et al. In search of the trauma memory: a meta-analysis of functional neuroimaging studies of symptom provocation in posttraumatic stress disorder (PTSD). PLOS ONE. 2013;8(3): e58150.

86. Shin LM, Davis FC, Vanelzakker MB, Dahlgren MK, Dubois SJ. Neuroimaging predictors of treatment response in anxiety disorders. Biol Mood Anxiety Disord. 2013;3(1):15.

87. Ziv M, Goldin PR, Jazaieri H, Hahn KS, Gross JJ. Emotion regulation in social anxiety disorder: behavioral and neural responses to three socio-emotional tasks. Biol Mood Anxiety Disord. 2013;3(1):20.

88. Courtin J, Bienvenu TCM, Einarsson EÖ, Herry C. Medial prefrontal cortex neuronal circuits in fear behavior. Neuroscience. 2013;240: 219-242.

89. Campbell-Sills L, Simmons AN, Lovero KL, Rochlin AA, Paulus MP, Stein MB. Functioning of neural systems supporting emotion regulation in anxiety-prone individuals. Neuroimage. 2011;54(1):689-696.

90. Liao W, Xu Q, Mantini D, et al. Altered gray matter morphometry and resting-state functional and structural connectivity in social anxiety disorder. Brain Res. 2011;1388:167-177.

91. Cha J, Greenberg T, Carlson JM, Dedora DJ, Hajcak G, Mujica-Parodi LR. Circuit-wide structural and functional measures predict ventromedial prefrontal cortex fear generalization: implications for generalized anxiety disorder. J Neurosci. 2014;34(11):4043-4053.

92. Chen Y, Fu K, Feng C, et al. Different regional gray matter loss in recent onset PTSD and non PTSD after a single prolonged trauma exposure. PLoS ONE. 2012;7(11):e48298.

93. Weber M, Killgore WDS, Rosso IM, et al. Voxel-based morphometric gray matter correlates of posttraumatic stress disorder. J Anxiety Disord. 2013;27(4):413-419.

94. Jovanovic T, Norrholm SD. Neural mechanisms of impaired fear inhibition in posttraumatic stress disorder. Front Behav Neurosci. 2011;5:44

95. Felmingham K, Williams LM, Kemp AH, et al. Neural responses to masked fear faces: sex differences and trauma exposure in posttraumatic stress disorder. J Abnorm Psychol. 2010;119(1):241-247.

96. Blair KS, Geraci M, Otero M, et al. Atypical modulation of medial prefrontal cortex to self-referential comments in generalized social phobia. Psychiatry Res. 2011;193(1):38-45.

97. Sripada CS, Angstadt M, Banks S, Nathan PJ, Liberzon I, Phan KL. Functional neuroimaging of mentalizing during the trust game in social anxiety disorder. Neuroreport. 2009;20(11):984-989.

98. Etkin A, Schatzberg AF. Common abnormalities and disorder-specific compensation during implicit regulation of emotional processing in generalized anxiety and major depressive disorders. Am J Psychiatry. 2011;168(9):968-978.

99. Price RB, Eldreth DA, Mohlman J. Deficient prefrontal attentional control in late-life generalized anxiety disorder: an fMRI investigation. Transl Psychiatry. 2011;1(10):e46.
100. Schlund MW, Verduzco G, Cataldo MF, Hoehn-Saric R. Generalized anxiety modulates frontal and limbic activation in major depression. Behav Brain Funct. 2012;8(1):8.

101. Lanius RA, Brewin CR, Bremner JD, et al. Does neuroimaging research examining the pathophysiology of posttraumatic stress disorder require medication-free patients? J Psychiatry Neurosci. 2010; 35(2):80-89.

102. Goldin P, Ziv M, Jazaieri H, Gross JJ. Randomized controlled trial of mindfulness-based stress reduction versus aerobic exercise: effects on the self-referential brain network in social anxiety disorder. Front Hum Neurosci. 2012;6:295.

103. Goldin PR, Ziv M, Jazaieri H, Hahn K, Heimberg R, Gross JJ. Impact of cognitive behavioral therapy for social anxiety disorder on the neural dynamics of cognitive reappraisal of negative self-beliefs: randomized clinical trial. JAMA Psychiatry. 2013;70(10):1048-1056.

104. Qiu C, Liao W, Ding J, et al. Regional homogeneity changes in social anxiety disorder: a resting-state fMRI study. Psychiatry Res. 2011; 194(1):47-53.

105. Woon FL, Sood S, Hedges DW. Hippocampal volume deficits associated with exposure to psychological trauma and posttraumatic stress disorder in adults: a meta-analysis. Prog Neuropsychopharmacol Biol Psychiatry. 2010;34(7):1181-1188.

106. Hettema J, Kettenmann B, Ahluwalia V, et al. Pilot multimodal twin imaging study of generalized anxiety disorder. Depress Anxiety. 2012;29(3):202-209.

107. Thomaes K, Dorrepaal E, Draijer N, et al. Increased anterior cingulate cortex and hippocampus activation in Complex PTSD during encoding of negative words. Soc Cogn Affect Neurosci. 2013;8(2):190-200.

108. Talati A, Pantazatos SP, Schneier FR, Weissman MM, Hirsch J. Gray matter abnormalities in social anxiety disorder: primary, replication, and specificity studies. Biol Psychiatry. 2013;73(1):75-84.

109. Brohawn KH, Offringa R, Pfaff DL, Hughes KC, Shin LM. The neural correlates of emotional memory in posttraumatic stress disorder. Biol Psychiatry. 2010;68(11):1023-1030.

110. Hayes JP, Vanelzakker MB, Shin LM. Emotion and cognition interactions in PTSD: a review of neurocognitive and neuroimaging studies. Front Integr Neurosci. 2012;6:89.

111. Schienle A, Scharmüller W, Leutgeb V, Schäfer A, Stark R. Sex differences in the functional and structural neuroanatomy of dental phobia. Brain Struct Funct. 2013;218(3):779-787.

112. Kreifelts B, Brück C, Ritter J, et al. They are laughing at me: cerebral mediation of cognitive biases in social anxiety. PLOS ONE. 2014; 9(6):e99815.

113. Offringa R, Handwerger Brohawn K, Staples LK, et al. Diminished rostral anterior cingulate cortex activation during trauma-unrelated emotional interference in PTSD. Biol Mood Anxiety Disord. 2013; 3(1): 10 .

114. Etkin A, Prater KE, Hoeft F, Menon V, Schatzberg AF. Failure of anterior cingulate activation and connectivity with the amygdala during implicit regulation of emotional processing in generalized anxiety disorder. Am J Psychiatry. 2010;167(5):545-554.

115. Baur V, Brühl AB, Herwig U, et al. Evidence of frontotemporal structural hypoconnectivity in social anxiety disorder: A quantitative fiber tractography study. Hum Brain Mapp. 2013;34(2):437-446.

116. Dodhia S, Hosanagar A, Fitzgerald DA, et al. Modulation of restingstate amygdala-frontal functional connectivity by oxytocin in generalized social anxiety disorder. Neuropsychopharmacology. 2014;39(9): 2061-2069.

117. Tromp DP, Grupe DW, Oathes DJ, et al. Reduced structural connectivity of a major frontolimbic pathway in generalized anxiety disorder. Arch Gen Psychiatry. 2012;69(9):925-934.

118. Strawn JR, Bitter SM, Weber WA, et al. Neurocircuitry of generalized anxiety disorder in adolescents: a pilot functional neuroimaging and functional connectivity study. Depress Anxiety. 2012;29(11):939-947.

119. Strawn JR, Wehry AM, Chu W-J, et al. Neuroanatomic abnormalities in adolescents with generalized anxiety disorder: a voxel-based morphometry study. Depress Anxiety. 2013;30(9):842-848. 
120. Roy AK, Fudge JL, Kelly C, et al. Intrinsic functional connectivity of amygdala-based networks in adolescent generalized anxiety disorder. J Am Acad Child Adolesc Psychiatry. 2013;52(3):290-299.e2.

121. Monk CS, Telzer EH, Mogg K, et al. Amygdala and ventrolateral prefrontal cortex activation to masked angry faces in children and adolescents with generalized anxiety disorder. Arch Gen Psychiatry. 2008;65(5):568-576.

122. Prater KE, Hosanagar A, Klumpp H, Angstadt M, Phan KL. Aberrant amygdala-frontal cortex connectivity during perception of fearful faces and at rest in generalized social anxiety disorder. Depress Anxiety. 2013;30(3):234-241.

123. Maslowsky J, Mogg K, Bradley BP, et al. A preliminary investigation of neural correlates of treatment in adolescents with generalized anxiety disorder. J Child Adolesc Psychopharmacol. 2010;20(2):105-111.
124. Liao W, Qiu C, Gentili C, et al. Altered effective connectivity network of the amygdala in social anxiety disorder: a resting-state FMRI study. PLoS ONE. 2010;5(12):e15238.

125. Greenberg T, Carlson JM, Cha J, Hajcak G, Mujica-Parodi LR. Ventromedial prefrontal cortex reactivity is altered in generalized anxiety disorder during fear generalization. Depress Anxiety. 2013;30(3):242-250.

126. Sripada RK, King AP, Garfinkel SN, et al. Altered resting-state amygdala functional connectivity in men with posttraumatic stress disorder. J Psychiatry Neurosci. 2012;37(4):241-249.

127. Frick A, Gingnell M, Marquand AF, et al. Classifying social anxiety disorder using multivoxel pattern analyses of brain function and structure. Behav Brain Res. 2014;259:330-335.

128. Anderson ML. Neural reuse: a fundamental organizational principle of the brain. Behav Brain Sci. 2010;33(4):245-266.

\section{Publish your work in this journal}

Therapeutics and Clinical Risk Management is an international, peerreviewed journal of clinical therapeutics and risk management, focusing on concise rapid reporting of clinical studies in all therapeutic areas, outcomes, safety, and programs for the effective, safe, and sustained use of medicines. This journal is indexed on PubMed Central, CAS,
EMBase, Scopus and the Elsevier Bibliographic databases. The manuscript management system is completely online and includes a very quick and fair peer-review system, which is all easy to use. Visit http://www.dovepress.com/testimonials.php to read real quotes from published authors.

Submit your manuscript here: http://www.dovepress.com/therapeutics-and-clinical-risk-management-journal 